\title{
Infrared Surveys for AGN
}

\author{
Harding E. Smith ${ }^{1}$ \\ Center for Astrophysics 6 Space Sciences and Department of Physics, \\ University of California, San Diego, La Jolla, CA 92093-0424, USA
}

\begin{abstract}
From the earliest extragalactic infrared studies AGN have shown themselves to be strong infrared sources, and IR surveys have revealed new populations of AGN. I briefly review current motivations for AGN surveys in the infrared and results from previous IR surveys. The Luminous Infrared Galaxies, which in some cases house dust-enshrouded AGN, submillimeter surveys, and recent studies of the cosmic x-ray and infrared backgrounds suggest that there is a population of highly-obscured AGN at high redshift. ISO Surveys have begun to resolve the infrared background and may have detected this obscured AGN population. New infrared surveys, particularly the SIRTF Wide-area Infrared Extragalactic Legacy Survey ( $S W I R E$ ) will detect this population and provide a platform for understanding the evolution of AGN, Starbursts and passively evolving galaxies in the context of large-scale structure and environment.
\end{abstract}

\section{Motivation: Active Galaxies in the Infrared}

There are a number of motivations for infrared studies of AGN and for carrying out surveys for AGN at infrared wavelengths:

1. AGN unification models require a dusty, molecular obscuring screen (torus?) which is expected to emit in the infrared.

2. Cosmic X-Ray Background models require a population of highly obscured AGN which may be detectable in the infrared.

3. Infrared/Starburst emission is a frequent companion to AGN activity; IR emission originates from dust which is either associated with the active nucleus (e.g. the PG QSO sample; Sanders et al. 1989) or with circumnuclear starburst emission as in the classical Seyfert galaxies NGC 1068 \& NGC 7469.

4. Luminous Infrared Galaxies frequently harbor AGN cores and may be a stage in the development of AGN from the merger of gas-rich galaxies.

\footnotetext{
${ }^{1}$ also, Infrared Processing and Analysis Center,Caltech/JPL, Pasadena, CA 91125
} 


\subsection{AGN Unification Models}

The AGN Unification models (Antonucci 1993) which have been popular for some time, and which have garnered considerable observational support, require an optically-thick, dusty obscuring screen, frequently assumed to be toroidal (Pier \& Krolik 1993; Granato \& Danese 1994), which obscures the central engine and broad emission-line region from equatorial lines of sight. In this scenario broad-line objects (Type 1 AGN - Sy1 galaxies and classical QSOs) are objects viewed near the pole of the torus, while narrow-line objects (Type 2 AGN Sy 2 galaxies) are viewed edge-on. Direct evidence for the existence of this obscuring screen is largely from studies of scattered broad-line emission in Sy 2 systems, but claims have been made that the mid-infrared spectra of some nearby galaxies match various torus models (Pier \& Krolik 1993; Granato \& Danese 1994). A critical point regarding this torus is that it will be optically thick at all wavelengths from soft $x$-rays through the mid-infrared. Even mid-infrared diagnostics may not reveal a highly obscured AGN. Hard X-rays and VLBI radio imaging are currently required to peer through the veil of obscuration.

\subsection{The Cosmic X-Ray and Infrared Backgrounds}

Recent models for the Cosmic X-Ray Background (CXB) require a population of highly obscured, perhaps even Compton-thick, AGN which emit primarily in the hard X-ray region in order to fit the X-ray background spectrum (Comastri et al. 1995; Gilli et al. 2001). This population increases in density with redshift. These AGN are expected to re-emit the absorbed radiation at infrared wavelengths providing a link between the CXB and the Cosmic InfraRed Background (CIRB). Recent measures of the CIRB with COBE (Hauser et al. 1998; Puget et al. 1996) show that more than half of the cosmic energy density (excluding the CMB) comes out in the infrared. An important question is then the relative importance of accretion energy due to AGN compared with stellar nucleosynthesis. Deep surveys with Chandra and XMM-Newton are resolving the CXB (Hornschemeier et al. 2001; Hasinger et al. 2001; Rosati et al. 2001) and ISO (Elbaz et al. 2002) has begun to resolve CIRB. New Surveys with SIRTF will convincingly determine the relationship between the faint X-ray and infrared populations revealing a great deal about the history of our Universe.

\subsection{Luminous Infrared Galaxies}

The most luminous galaxies in the Local Universe are Luminous Infrared Galaxies (LIGs) which emit the vast majority of their radiant power in the far-infrared between about $40-120 \mu \mathrm{m}$. These are gas-rich systems which are in the late stages of collisions or mergers. Extrapolation from the properties of lower luminosity Starburst galaxies suggests that the LIGs should be active star-forming systems (see Sanders \& Mirabel 1996 for a review). The LIGs also show many characterisatics of AGN and their luminosities reach values comparable to those of luminous QSOs. Much effort has been focused on whether LIGs are powered principally by Starburst or AGN activity, although both types of activity are almost certainly present. The discussion has been framed around a scenario proposed by Sanders et al. (1988) in which a merger of gas-rich disk galaxies stimulates a massive nuclear Starburst which in turn feeds a coalescing AGN core in the galaxy nucleus. As the AGN turns on, radiation pressure drives out 


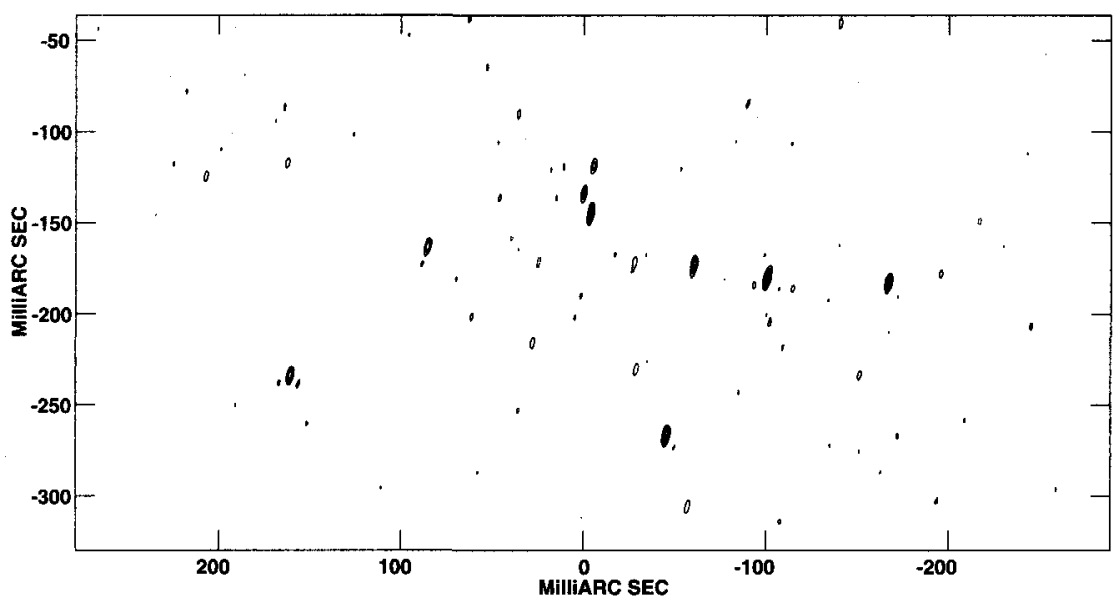

Figure 1. $18 \mathrm{~cm}$ VLBI image of the W Nucleus of Arp 220 from Smith et al. (1998). Over a dozen unresolved sources are interpreted as luminous radio supernovae in an intense starburst.

the shroud of dust, revealing a nascent quasar. The goal must be not only to understand the dominant source of energy in LIGs, but to understand the relationship between Starburst and AGN activity and other galaxy characteristics, and to place them into an evolutionary context.

We have for some time been using VLBI techniques to attempt to understand the power sources of LIGs and to place them in an evolutionary context. Analysis of a complete LIG sample (Smith, Lonsdale \& Lonsdale 1998) suggests that many LIGs may be interpreted as intense Starbursts like Arp 220 (Figure 1 ), but a nearly equal number must house AGN cores, as in the case of Mrk 231, whose nuclear structure implies a recent ignition $\left(t<<10^{6} y r\right)$ for AGN activity. Evidence is strong that a significant number of LIGs house obscured AGN activity and schematics are consistent with this Starburst-to-AGN scenario.

Of particular interest are the extremely luminous high- $z$ galaxies detected in recent SCUBA and other submillimeter surveys (Ivison et al. 2000). The SEDs of LIGs in the submillimeter have the unique characteristic that the positive K-correction offsets cosmological dimming for redshifts from $z \sim 1-10$ such that it is equally easy (or difficult) to detect infrared galaxies at $850 \mu \mathrm{m}$ over a range of high redshifts. Although only a handful of redshifts are available for submm sources owing to the large error circles for submm sources and the optical faintness of the small number of identified galaxies, existing redshifts confirm that the submm population lies at redshifts $1<z<3$. The existence of these sources implies a population of very luminous galaxies at very early epochs, with concomitant rapid evolution. The star-formation rates inferred from the luminosities of these systems are not easily produced in CDM galaxy formation models. In analogy with local LIGs, the submillimeter sources may be candidates for nascent QSOs at high-redshift. 


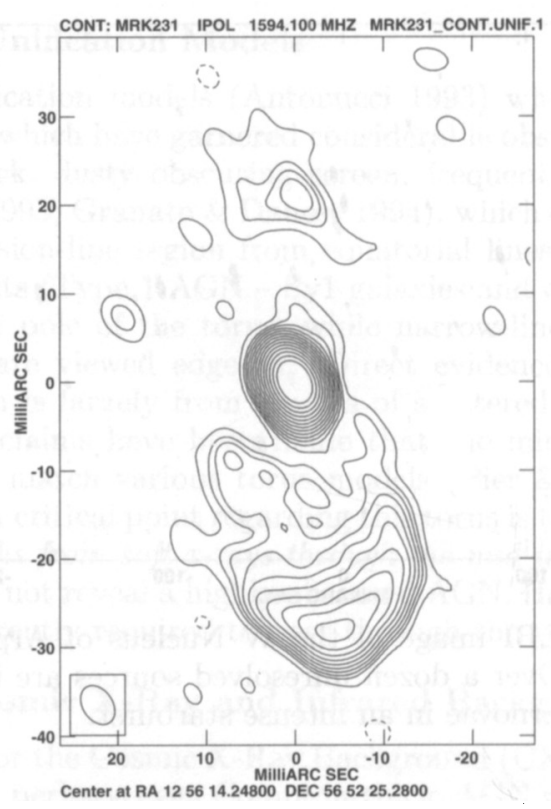

Figure 2. $18 \mathrm{~cm}$ VLBI image of the central 100pc of Mrk 231, interpreted as a nascent QSO; age $<<10^{6} \mathrm{yr}$.

\section{Active Galaxies with IRAS}

One of the most striking results from IRAS is that the infrared galaxy population evolves more rapidly than the optical galaxy population (Lonsdale et al. 1990) with a form similar to that for QSOs. Selection of Luminous IR Galaxies associated with FIRST radio sources (Stanford et al. 2001) or by FIR/Optical ratio (Smith et al. 2002; Lonsdale et al. 2002) have led to the detection of IRAS Faint Sources up to $z \sim 1$ with a small number of "hyperluminous" or lensed sources with redshifts in excess of 1 (e.g. Rowan-Robinson et al. 1991).

IRAS showed that the infrared spectra of QSOs are essentially similar, with a broad 'infrared bump' from about $2 \mu \mathrm{m}$ to $1 \mathrm{~mm}$, with a significant fraction $(10-50 \%)$ of the bolometric luminosity emitted at infrared wavelengths. This infrared emission is almost certainly thermal dust emission, but the location of this warm dust remains uncertain (Sanders et al. 1989) possibly associated with the outer edges of the accretion disk, the torus, or circumnuclear star formation.

Most of the known Sy 2 galaxies were discovered by IRAS, but the sensitivity of IRAS has limited our census of "Type 2 AGN" to $z<0.2$. As Padovani (1998) has previously stressed, at $z<0.2$ AGN Type 2 outnumber Type 1, suggesting that there are a large number of Sy 2 galaxies and Type 2 QSOs yet to be discovered at higher redshift. The expectation from our local census and from the CXB models suggests that an important task of future IR AGN Surveys will be detecting the high-redshift AGN 2 population, or explaining its absence in terms of AGN evolution. 


\section{2MASS Quasars}

The 2MASS AGN surveys have been reviewed by $\mathrm{R}$. Cutri (2002; this volume) and the characteristics of that population will be discussed only briefly. The surface density of 2MASS AGN is of the order of $0.5 \mathrm{deg}^{-2}$ with a space density comparable to that found in low-redshift optical-to-X-ray selected samples. The colors and polarization of 2MASS QSOs suggests that these are reddened rather than intrinsically red QSOs and the ratio of Type 1 AGN to Type 2 is about 2:1 - the obscured QSO 2's are not being found in 2MASS.

The color selection applied to detect 2MASS QSOs is $(J-K)>2$ and Cutri et al. argue that these QSOs are sufficiently red to have been missed in previous optical surveys. Analysis by the SDSS and 2MASS teams (Ivezic et al. 2002; this volume) suggests, however, that the incompleteness of the SDSS to such red QSOs is less than $10 \%$. The 2MASS AGN are faint in X-rays with a wide range in hardness-ratio (Wilkes et al. 2002) suggesting that the faintest and hardest sources may be highly obscured and could provide the missing X-ray population required to explain the $\mathrm{CXB}$.

\section{ISO AGN studies and Surveys}

\subsection{Mid-Infrared Diagnostics for AGN}

ISO has shown that mid-infrared spectroscopy is a powerful tool for source classification. In a series of papers (Lutz et al. 1998, Tran et al. 2001) the midinfrared molecular band strengths have been used to discriminate between AGNdominated $(7.7 \mu \mathrm{m}$ line to continuum $<1)$ and Starburst-dominated $(7.7 \mu \mathrm{m}$ to continuum $>1$ ) LIGs. These results suggest that the majority of LIGs with $\log L_{\text {fir }}<12\left(L_{\odot}\right)$, the "ULIGs", are Starburst-dominated, with an increasing AGN-fraction with increasing $L_{\text {fir }}$. Comparison with optical spectroscopy (Lutz, Veilleux \& Genzel 1999) shows that the infrared classifications are consistent with classical optical excitation methods and suggests that infrared galaxies with $L I N E R$ spectra are Starbursts (Veilleux 2002; this volume).

A somewhat different direction has been taken by Clavel et al. (2000) who compared the strengths of the mid-infrared bands among AGN types. In their sample there is a clear distinction between Sy 1 galaxies, showing low $W_{\lambda}(7.7 \mu \mathrm{m})$, and Sy 1.5-2 galaxies with higher values of $W_{\lambda}(7.7 \mu \mathrm{m})$. The $7.7 \mu \mathrm{m}$ luminosities of the Sy galaxy types are comparable, however. They interpret this result in terms of the classical AGN-torus model, suggesting that the warm AGN continuum from the inner torus is extinguished in the edge-on Sy 2 systems but visible in the Sy 1 systems, viewed face-on, whereas the extended, Starburstrelated mid-infrared features are visible in both types of galaxies.

Taken together these results underscore the caveat discussed above, that AGN activity in compact, highly-obscured infrared galaxies may remain hidden even at mid-infrared wavelengths.

\subsection{ISO Deep Surveys}

A number of deep surveys were undertaken with the ISO satellite at wavelengths of $7,15,90$, and $170 \mu \mathrm{m}$. These are reviewed in detail by Taniguchi (2002; this 
volume) and the results will only be summarized here. The principal result from these surveys is the continuing high surface densities of infrared galaxies as ISO pushed to lower flux-densities - as low as $\sim 10 \mu \mathrm{Jy}$ at the lower wavelengths - requiring continued steep evolution with redshift. There are a number of models with varying prescriptions for the evolving population (Franceschini et al. 2001; Rowan-Robinson 2001; Xu et al. 2001). The models of Xu et al., based upon a local complete $24 \mu \mathrm{m}$ sample, require luminosity evolution rates as high as $L \propto(1+z)^{4.2}$ combined with density evolution, $\rho \propto(1+z)^{2}$, up to $z \sim 1$ for the Starburst population to match the $\log N-\log S$ relation; the number of LIGs at $z \sim 1$ is thus estimated to be approximately 40 times higher than in the local Universe (Franceschini et al. 2001). The Xu et al. model has pure luminosity evolution for the AGN population, $L \propto(1+z)^{3.5}$, whereas the "normal" galaxy population evolves as $L \propto(1+z)^{1.5}$.

With about $2000+$ galaxies resolved by ISO and over 400 spectroscopic observations, AGN account for about $10 \%$ of the identified sources in the ISO Surveys. The ratio of AGN type 1 to type 2 in these surveys is about unity, at variance with the IRAS results at higher flux-density.

Elbaz et al. (2002) have analyzed the deep ISO $15 \mu \mathrm{m}$ counts and estimated that the IR galaxies detected by ISO to a $15 \mu \mathrm{m}$ flux-density, $S_{15 \mu m}>50 \mu \mathrm{Jy}$, contribute over half of the CIRB and that AGN may contribute, at most about $20 \%$ of the IR background. Fadda et al. (2002) have combined the deep ISOCAM/XMM-Newton data from the HDFN and Lockman Hole with the brighter ELAIS S1/BeppoSAX data to estimate the AGN contribution to midIR surveys and the CIRB. Using X-ray emission as an indicator of AGN activity, Fadda et al. estimate that $15-20 \%$ of the mid-infrared emission in Lockman and HDFN originates from AGN. The detection (AGN) fraction of $15 \mu \mathrm{m}$ sources increases with X-ray energy from $30 \%$ below $2 \mathrm{keV}$ to over $60 \%$ above $5 \mathrm{keV}$, as might be expected if the X-ray background is produced by obscured, highcolumn sources. Again, the fraction of the CIRB attributed to AGN-accretion energy is estimated to be less than $20 \%$.

Further analysis of red X-ray luminous galaxies $\left(L_{x} \sim 10^{43}-10^{45} \mathrm{erg} / \mathrm{s}\right)$ in the same sample (Franceschini et al. 2002) suggests that these may be highlyobscured AGN as predicted by CXB background models. The mid-infrared SEDs of these sources are well reproduced by model spectra of obscured QSOs with $\tau_{0.3 \mu m} \sim 30-40$ and the ratio of Type 1-to-Type 2 AGN is 1:3 in agreement with predictions.

The areas surveyed remain small, less than a few hundred square arcminutes to $S_{15 \mu m}<100 \mu \mathrm{Jy}$, and statistics are restricted to small numbers with a few tens of confirmed AGN. The lower sensitivities at the longer wavelengths require the above analyses to employ template SEDs for estimating the contributions of ISO sources at the peak of CIRB near $140 \mu \mathrm{m}$. With the wide range of mid-tofar-IR SEDS observed in the local Universe (e.g. the Far-IR/Mid-IR ratio may differ by an order of magnitude between a "typical Starburst" like M82 and a LIG such as Arp 220) these intriguing results remain tentative. 


\section{SWIRE: The SIRTF Wide-area InfraRed Extragalactic Survey}

For SIRTF, the last of its Great Observatories, NASA has selected a set of Legacy Programs, designed to be major surveys of general interest to the Astronomical community and to be carried out in the first year of the SIRTF mission. The Survey data will be distributed to the community in time for use in preparation of General Observer Proposals with no proprietary period for Legacy data.

The SIRTF Wide-area InfraRed Extragalactic Survey (SWIRE, Dr. Carol Lonsdale, P.I.) is the largest of the six SIRTF Legacy Surveys (851 hours), surveying approximately 67 square degrees in all 7 SIRTF imaging bands. A current description of the SWIRE Survey is given on the SWIRE WebPages: http://www.ipac.caltech.edu/SWIRE. Table 1 lists the Survey sensitivities.

Table 1. SWIRE Sensitivity Limits (est. $5 \sigma$ )

\begin{tabular}{rrcrrc}
\hline & IRAC & & \multicolumn{3}{c}{ MIPS } \\
$\lambda$ & Sensitivity & Resolution & $\lambda$ & Sensitivity & Resolution \\
\hline $3.6 \mu \mathrm{m}$ & $7.3 \mu \mathrm{Jy}$ & $0.9^{\prime \prime}$ & $24 \mu \mathrm{m}$ & $0.45 \mathrm{mJy}$ & $5.5^{\prime \prime}$ \\
$4.5 \mu \mathrm{m}$ & $9.7 \mu \mathrm{Jy}$ & $1.2^{\prime \prime}$ & $70 \mu \mathrm{m}$ & $2.75 \mathrm{mJy}$ & $16^{\prime \prime}$ \\
$5.8 \mu \mathrm{m}$ & $27.5 \mu \mathrm{Jy}$ & $1.5^{\prime \prime}$ & $160 \mu \mathrm{m}$ & $17.5 \mathrm{mJy}$ & $36^{\prime \prime}$ \\
$8.0 \mu \mathrm{m}$ & $32.5 \mu \mathrm{Jy}$ & $1.8^{\prime \prime}$ & & & \\
\hline \hline
\end{tabular}

The Survey will cover seven high-latitude fields, selected to be the most transparent, lowest background fields in the sky. The fields, covering between 5 and 15 sq. deg. include previously well-known IR extragalactic survey fields (e.g. Lockman and the ELAIS ISO Survey Fields) and x-ray fields (Chandra Deep South and XMM Large Scale Survey) are shown in Table 2.

Table 2. SWIRE Survey Fields

\begin{tabular}{lccrl}
\hline Field & \multicolumn{2}{c}{ Center (J200) } & $\begin{array}{r}\text { Area } \\
\text { RA }\end{array}$ & $\begin{array}{l}\text { Background } \\
\text { Dec }\end{array}$ \\
\hline ELAIS S1 & $00^{h} 38^{m} 30^{s}$ & $-44^{\circ} 00^{\prime}$ & 14.8 & 0.42 \\
XMM-LSS & $02^{h} 21^{m} 00^{s}$ & $-05^{\circ} 00^{\prime}$ & 9.3 & 1.3 \\
Chandra-S & $03^{h} 32^{m} 00^{s}$ & $-28^{\circ} 16^{\prime}$ & 7.2 & 0.46 \\
Lockman & $10^{h} 45^{m} 00^{s}$ & $+58^{\circ} 00^{\prime}$ & 14.8 & 0.38 \\
Lonsdale & $14^{h} 41^{m} 00^{s}$ & $+59^{\circ} 25^{\prime}$ & 6.9 & 0.47 \\
ELAIS N1 & $16^{h} 11^{m} 00^{s}$ & $+55^{\circ} 00^{\prime}$ & 9.3 & 0.44 \\
ELAIS N2 & $16^{h} 36^{m} 48^{s}$ & $+41^{\circ} 02^{\prime}$ & 4.5 & 0.42 \\
\hline \hline
\end{tabular}

The SWIRE science goal is to enable fundamental studies of galaxy evolution in the infrared for $0.5<z<3$ :

- Evolution of star-forming and passively evolving galaxies in the context of structure formation and environment.

- Spatial distribution and clustering of evolved galaxies, Starbursts, \& AGN.

- The evolutionary relationship between galaxies and AGN and the contribution of AGN accretion energy to the cosmic backgrounds. 
Galaxy evolution models which match the IRAS/ISO galaxy counts at all wavelengths from $7-100 \mu \mathrm{m}$ as well as the CIRB (Xu et al. 2001) predict that SWIRE will detect of the order of 2 million galaxies - spheroids and evolved stellar systems with IRAC, and active star-forming systems with MIPS. SWIRE will also detect about 25,000 classical AGN, and an unknown number, perhaps several times as many, dust-enshrouded AGN.

Recent estimates of the "Universal Star-formation History $(S F H)$ " (Steidel et al. 1999) suggest that the bulk of cosmic evolution occurs between redshifts, $0.5<z<3$, the redshift interval for which SWIRE is optimized. The median redshift is predicted to be, $\langle z\rangle \sim 1$, where many estimates find a peak in the $S F H$; luminous infrared galaxies will be detected by SWIRE out to $z \sim 3$. Previous estimates of the $S F H$ have varying, frequently large and uncertain corrections for extinction. SWIRE will directly measure the total star-formation rates as a function of redshift and environment over this critical range of time and redshift.

A key element in the SWIRE Survey design is to enable galaxy evolution studies in the context of large-scale structure/environment. One of the SWIRE Survey fields covers the deep survey areas of the XMM-LSS Survey (Pierre 2001) so that the infrared galaxy census may be directly tied to the presence of rich $\mathrm{X}$-ray clusters to $z>1$. SWIRE will sample several hundred, $100 \mathrm{Mpc}$ scale co-moving volume cells enabling a variety of large-scale structure measures from correlation functions, power spectra, and counts-in-cells to direct comparison with model calculations. SWIRE's measures of the star-formation as a function of environment will be important input for CDM simulations which have been exceedingly successful in simulating the development of structure in the early Universe, but perhaps less so in simulating galaxy evolution within that structure owing to the complexity of the physics of star formation (e.g. Kay et al. 2002).

Of more direct importance to this Conference, the similarities of AGN SEDs in the mid-far Infrared suggests that SWIRE will be unbiased with respect to AGN types and ages, enabling a complete census of AGN out to redshifts greater than 1 . Although the detection rates should be unbiased, the similarity between the SEDs of obscured AGN to those of Starbursts, and the extreme optical depths will make identifying the obscured AGN population very challenging. Low-frequency radio surveys will, of course, identify radio-loud AGN, but these make up only $10-15 \%$ of the AGN population. For this reason the XMM-LSS Survey, along with current and planned deeper surveys in hard X-rays will be vital to identifying SWIRE AGN.

\subsection{Supporting Observations}

An aggressive program of ground-based optical, near-infrared and radio observations is planned in support of the SWIRE Survey and we are actively pursuing other programs with HST, Chandra, XMM and Galex. As already described Chandra and XMM Surveys will be important for discovering the obscured AGN population, if it exists. SWIRE has entered into cooperation with the Galex team so that the SWIRE fields will be included in the Galex Deep Survey.

The SWIRE Optical-Near Infrared goal is to obtain moderate-depth optical multi-band $\left(g^{\prime} \sim 25.7, r^{\prime} \sim 25, i^{\prime} \sim 24\right.$; Vega magnitudes, $5 \sigma$ detection for a $2^{\prime \prime}$ galaxy) data for the entire Survey area. At these limits we expect to detect approximately $2 / 3$ of SWIRE sources detected by both MIPS and IRAC. The 
ELAIS N1, N2 fields have already been imaged to somewhat shallower limits $\left(r^{\prime} \sim 24\right)$ as part of the INT Wide Field Survey and efforts continue to push deeper in the optical and into the near infrared as part of the UK SWIRE Program (ISLES and UKIDSS projects respectively). An extensive program for observations of ELAIS S1 is being undertaken at ESO. Optical and near-infrared imaging of the Lockman, Lonsdale and CDFS fields are being undertaken at KPNO and CTIO with the Mosaic cameras and FLAMINGOS infrared imager.

Two major SWIRE radio surveys are planned. The median $20 \mathrm{~cm}$ flux density predicted for SWIRE Starburst galaxies is $\sim 43 \mu \mathrm{Jy}$ - to faint to survey the entire area to this depth. We have therefore planned a deep pencil-beam VLA Survey (F. Owen, PI) and an extended shallow VLA survey (J. Condon, PI):

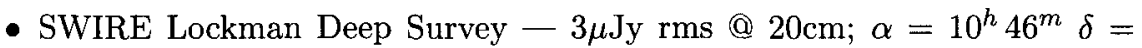
$+59^{\circ} 01^{\prime} ; 30^{\prime}$ VLA primary beam. The Deep VLA Survey is nearly completed and data analysis is just beginning.

- Cosmic Windows VLA Survey - 50 $\mu \mathrm{Jy} \mathrm{rms} @ 20 \mathrm{~cm}$ in the combined fields of SWIRE, Galex and XMM-LSS which are accessible to the VLA. This Survey is being proposed for the next VLA large survey program.

\section{Benediction}

The SWIRE Legacy Survey is a community Survey; the large dataset which is being accumulated reflects the synergies which between the Legacy program and other community surveys. With a couple of million galaxies and several tens of thousands of AGN, many with redshift estimates and SEDs from x-ray to radio, the SWIRE database will be released to the community through IPAC's Infrared Science Archive. We hope that SWIRE will provide a rich datamine for the entire community and will provide answers to many of the questions posed here. If you have projects that you would like to do with $S W I R E$ data, please visit the SWIRE WebPages and/or contact one of the team members.

It is a pleasure to thank the local organizers, especially Areg Mickaelian and Ed Khachikian, and the scientific organizing committee for my second stimulating visit to beautiful Armenia. This research was supported by the US NASA.

\section{References}

Antonucci, R. 1993, ARA\&A, 31, 473.

Clavel et al. 2000, A\&A, 357, 839.

Comastri, A., Setti, G., Zamorani, G., \& Hasinger, G. 1995, A\&A, 296, 1.

Elbaz, D., et al. 2002, A\&A, in press. (astro-ph/0201328)

Fadda, D., et al. 2002, A\&A, in press. (astro-ph/0111412)

Franceschini, A., et al. 2002, A\&A, in press. (astro-ph/0111413).

Franceschini, A., et al. 2001, A\&A, 378, 1.

Gilli, R., Salvati, M. \& Hasinger, G. 2001, A\&A, 366, 407.

Granato, G. \& Danese L., 1994, MNRAS, 268, 235.

Hasinger, G., et al. 2001 A\&A, 365, 45. 
Hauser, M., et al. 1998, ApJ, 508, 25.

Hornschemeier, A., et al. 2001, ApJ, 554, 742.

Ivison, R., et al., 2000, MNRAS, 315, 209.

Kay, S., Pearce, F., Frenk, C., \& Jenkins, A. 2002, MNRAS, 330, 113.

Lonsdale, C. J, et al. 1990, ApJ, 358, 60.

Lonsdale, C., Hurt, R., \& Smith, H. E., \& Xu, C. 2002, ApJ, in preparation.

Lutz, D., et al. 1998, ApJ, 505, L103.

Lutz, D., Veilleux, S. \& Genzel, R. 1999, ApJ, 517, L13.

Padovani, P. 1998 in New Horizons from Multi-Wavelength Sky Surveys, ed. B. McLean, D. Golombek, J. Hayes, \& H. Payne, (Kluwer), p. 257.

Pier, E. \& Krolik, J. 1993, ApJ, 418, 673.

Pierre, M. 2001 in Where's the Matter?, eds. L. Tresse \& M. Treyer, in press. (astro-ph/0111242)

Puget, J-L., et al. 1996, A\&A, 308, 5.

Rosati, P. et al. 2002, ApJ, 566, 667.

Rowan-Robinson. M., et al. 1991, Nature, 351, 719.

Rowan-Robinson, M. 2001, ApJ, 549, 745.

Sanders, D. B. \& Mirabel, I. F. 1996, ARA\&A, 34, 749.

Sanders, D., et al. 1988, ApJ, 325, 74.

Sanders, D., et al. 1989, ApJ, 347, 29.

Smith, H. E., Lonsdale, C., \& Lonsdale, C. 1998, ApJ, 492, 137.

Smith, H. E., Lonsdale, C., Lonsdale, C. \& Diamond, P. 1998, ApJ, 493, L17.

Smith, H. E., Lonsdale, C., Hurt, R. \& Siana, B. 2002, ApJ, in preparation.

Stanford, S., et al. 2000, ApJS, 131, 185.

Steidel, C, et al. 1999, ApJ, 519, 1.

Tran, Q., et al. 2001, ApJ, 552, 527.

Wilkes, B., et al. 2002, ApJ, in press. (astro-ph/0112433)

Xu, C., Lonsdale, C., Shupe, D., O'Linger, J., \& Masci, F. 2001, ApJ, 562, 179. 